\title{
Primary cerebral rhabdomyosarcoma - an oncological headache
}

\author{
Niranjan Vijayaraghavan ${ }^{1}$, John Mohan Mathew ${ }^{2}$ \\ ${ }^{1}$ Department of Medical Oncology, Madras Medical College, Tamilnadu, India \\ ${ }^{2}$ Department of Radiation Oncology, Regional Cancer Centre, Trivandrum Kerala, India
}

A 23-year-old female presented with complaints of progressive headache and vomiting of two months duration and first episode of GTCS. There was no significant medical or family history, no history of alcohol intake or substance abuse. On examination she was drowsy but responding to commands. Neurological examination revealed left sided hemiparesis with no other deficits. Blood tests after admission revealed no abnormalities. MRI imaging of brain revealed a $7.5 \times 6.0 \times 6.5 \mathrm{~cm}$ sized well-defined T1 hypo and T2 hyper-intense cystic lesion with septations in the right frontoparietal region along with an eccentrically located enhancing solid component (Fig. 1). Correlating

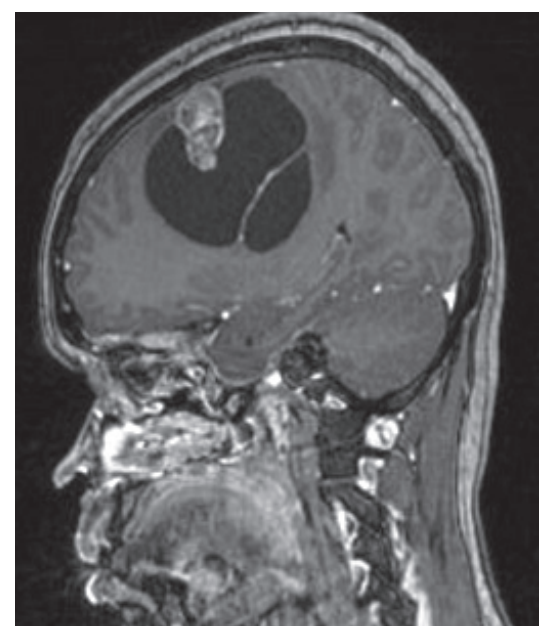

Figure 1. T1 contrast MRI image shows hypointense cystic lesion with enhancing intramural nodule the clinical features and imaging findings, a working diagnosis of CNS tumor was made and she underwent maximal safe resection. Postoperative histopathology showed spindle shaped tumor cells along with cells of rhabdoid morphology. IHC was positive for GFAP, Desmin (Fig. 2), Myo D1 and negative for EMA which was suggestive of rhabdomyosarcoma which was non-meningeal in origin. MRI imaging of the head and neck, thorax, abdomen, pelvis and extremities did not reveal any other lesion. Bone scan and bone marrow biopsy and aspirate was negative for metastasis. Thereby, a final diagnosis of primary cerebral rhabdomyo-

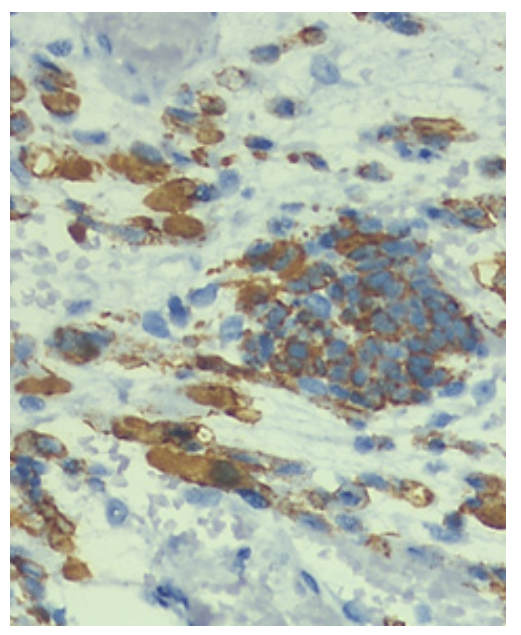

Figure 2. Section shows strong positivity of the rhabdoid tumor cells for Desmin. Immunohistochemistry with DAKO primary antibody, Diaminobenzidine stain, $\times 400$ (black arrow)

Address for correspondence: Dr. John M. Mathew MD DNB Senior Resident, Department of Radiation Oncology, Regional Cancer Centre, Trivandrum, Kerala, India, tel: +91 9809033937; e-mail: johnmathai99@gmail.com

This article is available in open access under Creative Common Attribution-Non-Commercial-No Derivatives 4.0 International (CC BY-NC-ND 4.0) license, allowing to download articles and share them with others as long as they credit the authors and the publisher, but without permission to change them in any way or use them commercially 
sarcoma (RMS) was made. Following this, she was treated with adjuvant radiation therapy to a dose of 60 Gy by the VMAT (Volumetric modulated arc therapy) technique followed by 6 cycles of chemotherapy with VAC regimen (Vincristine, Actinomycin D and Cyclophosphamide). Post chemo imaging done after 3 months was suggestive of progressive disease and patient expired 14 months after diagnosis.

Primary Intracranial RMS are rare and mostly seen in the pediatric population, with the posterior cranial fossa being the most common location [1]. In general, it is believed that RMS arises from a population of embryonic pericapillary mesenchymal cells which persist after birth and retain the ability to differentiate along a number of pathways [2].

From a neuro-radiological point of view, RMS doesn't have unique radiological features that differentiate it from other malignant brain tumors. The tumor usually exhibits homogenous/intermediate signal intensity with contrast enhancement on $\mathrm{T} 1$ and high signal intensity on T2-weighted images [2]. The rarity of primary CNS RMS does not allow a conclusive therapeutic regimen. The extent of postoperative residual disease is the most important prognostic factor and rapid recurrence after surgical resection is usual [3].

Among the adult primary cerebral RMS reported in literature with survival longer than a year, almost all cases received radiation dose of $60 \mathrm{~Gy}[2,4,5]$. This and the clinical behavior of the tumour, akin to that of high grade glioma formed the rationale of treating the patient to a dose of $60 \mathrm{~Gy}$. The chemotherapy regimen used was extrapolated from the treatment of systemic RMS where VAC based regimen is usually given for 2 years [4].
In spite of aggressive multimodality treatment the prognosis of primary CNS RMS is poor with a median survival after diagnosis of 7 months [1]. Treatment with a radiation dose of $60 \mathrm{~Gy}$ and the delivery of 6 cycles of triple agent chemo could have contributed to the 14-month survival seen after diagnosis in this patient.

\section{Conflict of interest}

None declared.

\section{Funding}

None declared.

\section{References}

1. Tomei G, Grimoldi N, Cappricci E, et al. Primary intracranial rhabdomyosarcoma: report of two cases. Childs Nerv Syst. 1989; 5(4): 246-249, doi: 10.1007/BF00271028, indexed in Pubmed: 2790836.

2. Celli P, Cervoni L, Maraglino C. Primary rhabdomyosarcoma of the brain: observations on a case with clinical and radiological evidence of cure. J Neurooncol. 1998; 36(3): 259-267, doi: 10.1023/a:1005884202389, indexed in Pubmed: 9524104.

3. Yagishita S, Itoh Y, Chiba Y, et al. Primary rhabdomyosarcoma of the cerebrum. Acta Neuropathol. 1979; 45(2): 111-115, doi: 10.1007/bf00691888, indexed in Pubmed: 419933.

4. Pirillo V, Cipriano Cecchi P, Tripodi M, et al. Primary cerebral alveolar rhabdomyosarcoma in adult. Rare Tumors. 2011; 3(2): e26, doi: 10.4081/rt.2011.e26, indexed in Pubmed: 21769325.

5. Desai KB, Mella D, Pan E. An Adult Patient With Rare Primary Intracranial Alveolar Rhabdomyosarcoma. Anticancer Res. 2019; 39(6): 3067-3070, doi: 10.21873/ anticanres.13441, indexed in Pubmed: 31177150.

6. Crist WM, Garnsey L, Beltangady MS, et al. Prognosis in children with rhabdomyosarcoma: a report of the intergroup rhabdomyosarcoma studies I and II. Intergroup Rhabdomyosarcoma Committee. J Clin Oncol. 1990; 8(3): 443-452, doi: 10.1200/JCO.1990.8.3.443, indexed in Pubmed: 2407808. 